\title{
ESTIMATING MODULI AND FRICTION ANGLE FROM SELF-BORING PRESSUREMETER TESTS IN SAND
}

\author{
RAMESH C. GUPTA ${ }^{\mathrm{i})}$
}

\begin{abstract}
Self-boring pressuremeter test (SBPMT) data has been analyzed based on cylindrical cavity expansion method and dimensionless cylindrical cavity expansion factors $\left(F^{\prime \prime}{ }_{\mathrm{q}}\right)$ to determine angle of friction, rigidity index $\left(I_{\mathrm{r}}\right)$ and modulus of elasticity $(E)$. Then, based on a hyperbolic model, secant modulus $\left(E_{\mathrm{sf}}\right)$, secant modulus at 50 percent failure stresses $\left(E_{50}\right)$, and initial modulus $\left(E_{\mathrm{i}}\right)$ have been determined. Reasonable values of angle of friction, initial modulus $\left(E_{\mathrm{j}}\right)$, and secant modulus of deformation at failure $\left(E_{\mathrm{sf}}\right)$ have been determined for sand deposits located in different geographical regions. The secant modulus $\left(E_{50}\right)$ determined by this method compares well with unload-reload modulus, $E_{\mathrm{r}}$, determined from unload-reload cycle of the SBPM tests.
\end{abstract}

Key words: expansion, friction angle, pressuremeter test, sand, Young's modulus (IGC: E2/E4)

\section{INTRODUCTION}

Self-boring pressuremeter (SBPM) has been used widely to determine soil properties of sand. The main advantage of the SBPM is that it can be installed with minimized initial disturbance, and therefore provides tests and geotechnical parameters of sands in almost undisturbed conditions, and in their natural environment. When it is expanded, it simulates the cylindrical cavity expansion from a finite radius; with this view, Gupta (2005) performed a finite strain analysis to determine volumetric strains in the plastic zone using cylindrical cavity expansion from finite radius so that an analysis of SBPMT data could be performed accurately. This analysis is based on Vesic's cavity expansion theory (Vesic, 1972), which assumes a linear stress-strain relationship during expansion of cavity from beginning of expansion to the stage when the state of equilibrium is reached at the face of cavity. On this basis, a method of determining angle of friction $(\phi)$ and modulus of elasticity $(E)$, by a technique of matching dimensional cavity expansion factor $\left(F^{\prime \prime}{ }_{q}\right)$ versus circumferential strain $\left(\varepsilon_{\theta \theta}\right)$ curve obtained from a SBPM test with that obtained from the theoretical analysis at selected values of $\phi$ and $E$, has been introduced (Gupta, 2005). Here it may be noted that $F^{\prime \prime}{ }_{9}$ is equal to internal cavity pressure, $p(t)$, applied during a SBPM test at any instant of time, divided by effective horizontal stress $\left(\sigma^{\prime}{ }_{\mathrm{h}}\right)$, measured during the SBPM test. However, real soils exhibit nonlinear stress-strain relationship even in the elastic state (Duncan and Chang, 1970; Mayne, 2001). Therefore, it becomes necessary to examine how the above method can be used to create a nonlinear stress-strain model or in other words how this method can be expanded to determine secant modulus at failure $\left(E_{\mathrm{sf}}\right)$, secant modulus at $50 \%$ failure stresses $\left(E_{50}\right)$, and initial tangent modulus $\left(E_{\mathrm{i}}\right)$. In geotechnical design and practice, $E_{50}$ and $E_{\mathrm{i}}$ are frequently used to determine settlement related problems, and stress distribution under footings in working stress conditions. In this paper, a method has been introduced to determine, $E_{\mathrm{sf}}, E_{50}$ and $E_{\mathrm{i}}$ from SBPMT data, after completing the analysis given in the companion paper (Gupta, 2005).

\section{HYPERBOLIC NONLINEAR STRESS-STRAIN MODEL}

Results of the triaxial compression tests and screw plate load tests have shown that the stress-strain relationship of sand is nonlinear. It is quite difficult to determine the initial modulus $\left(E_{\mathrm{i}}\right)$ accurately from such tests, since the slope of the stress-strain curve changes rapidly even at very small strains. The hyperbolic model (Kondner, 1963; Duncan and Chang, 1970; Desai and Christian, 1977), which was used for triaxial compression tests, can also be used to approximate the nonlinear stress-strain curves for cylindrical cavity expansion, as described below:

At the face of cavity, prior to reaching state of equilibrium, radial displacement $\left(\xi_{\text {rc }}\right)$ and circumferential strain, $\left(\varepsilon_{\theta \theta}\right)$ are given by (Baguelin, 1978):

$$
\xi_{\mathrm{rc}}=\frac{1+v}{E} R(t)\left(p(t)-\sigma_{\mathrm{m}}\right)
$$

i) Ph.D., Senior Geotechnical Engineer, Structure \& Bridge Div., Virginia Department of Transportation, 1401 East Broad St., Richmond, VA 23219, USA (ramesh.gupta@vdot.virginia.gov).

The manuscript for this paper was received for review on August 27, 2004; approved on July $20,2005$.

Written discussions on this paper should be submitted before May 1, 2006 to the Japanese Geotechnical Society, 4-38-2, Sengoku, Bunkyo-ku, Tokyo 112-0011, Japan. Upon request the closing date may be extended one month. 


$$
\varepsilon_{\theta \theta}=\frac{1+v}{E}\left(p(t)-\sigma_{\mathrm{m}}\right)=\frac{1+v}{2 E}\left(\sigma_{\mathrm{rr}}-\sigma_{\theta \theta}\right)
$$

It may be noted that in elastic phase of cavity expansion, $\varepsilon_{\theta \theta}$ is equal to $-\varepsilon_{\mathrm{rr}}$, where $\varepsilon_{\mathrm{rr}}$ is radial strain. When state of equilibrium has taken place at the face of cavity, peak stresses are related by (Vesic, 1972; Gupta, 2005):

$$
\left(\sigma_{\mathrm{rr}}-\sigma_{\theta \theta}\right)_{\mathrm{f}}=2 q^{\prime} \sin \phi=2 \sigma_{\mathrm{m}}^{\prime} \sin \phi
$$

Prior to reaching a state of equilibrium at the face of cavity, nonlinear stress-strain hyperbolic model for cylindrical cavity expansion can be represented by:

$$
\left(\sigma_{\mathrm{rr}}-\sigma_{\theta \theta}\right)=\frac{\varepsilon_{\theta \theta}}{b+a \varepsilon_{\theta \theta}} \text { or } \frac{\varepsilon_{\theta \theta}}{\left(\sigma_{\mathrm{rr}}-\sigma_{\theta \theta}\right)}=b+a \varepsilon_{\theta \theta}
$$

At the initial beginning, strains are small, even less than $10^{-4}$ percent, such as found during wave propagation, and therefore, at that instant, the product $a \varepsilon_{\theta \theta}$ becomes very small and can be neglected; then Eq. (4) becomes:

$$
\frac{\varepsilon_{\theta \theta}}{\left(\sigma_{\mathrm{rr}}-\sigma_{\theta \theta}\right)}=b \text { or } \frac{\left(\sigma_{\mathrm{rr}}-\sigma_{\theta \theta}\right)}{\varepsilon_{\theta \theta}}=\frac{1}{b}=E_{\mathrm{i}}
$$

$E_{\mathrm{i}}$ is the initial modulus. Substituting value of $b$ from Eq. (5) in Eq. (4), nonlinear stress-strain relationship becomes:

$$
\frac{\varepsilon_{\theta \theta}}{\left(\sigma_{\mathrm{rr}}-\sigma_{\theta \theta}\right)}=\frac{1}{E_{\mathrm{i}}}+a \varepsilon_{\theta \theta}
$$

At very large strains, the product $a \varepsilon_{\theta \theta}$ becomes very large compared to $1 / E_{\mathrm{i}}$, and therefore, at that instant, $1 / E_{\mathrm{i}}$ can be neglected, then, Eq. (6) becomes:

$$
\frac{\varepsilon_{\theta \theta}}{\left(\sigma_{\mathrm{rr}}-\sigma_{\theta \theta}\right)}=a \varepsilon_{\theta \theta} \text { or } \frac{1}{a}=\left(\sigma_{\mathrm{rr}}-\sigma_{\theta \theta}\right)_{\mathrm{asymp}}
$$

Where $1 / a$ or $\left(\sigma_{\mathrm{rr}}-\sigma_{\theta \theta}\right)_{\text {asymp }}$ in Eq. (7) represents asymptotic value of the hyperbolic model. Substituting this value of ' $a$ ' in Eq. (6), and rearranging terms, hyperbolic model representing the nonlinear stress-strain relationship is given by:

$$
\left(\sigma_{\mathrm{rr}}-\sigma_{\theta \theta}\right)=\frac{\varepsilon_{\theta \theta}}{\frac{1}{E_{\mathrm{i}}}+\frac{\varepsilon_{\theta \theta}}{\left(\sigma_{\mathrm{rr}}-\sigma_{\theta \theta}\right)_{\mathrm{asymp}}}}=\frac{\varepsilon_{\theta \theta}}{\frac{1}{E_{\mathrm{i}}}+\frac{\varepsilon_{\theta \theta} R_{\mathrm{f}}}{\left(\sigma_{\mathrm{rr}}-\sigma_{\theta \theta}\right)_{\mathrm{f}}}}
$$

Since the compressive strength or peak stresses, $\left(\sigma_{\mathrm{rr}}-\right.$ $\left.\sigma_{\theta \theta}\right)_{\mathrm{f}}$ during expansion of cavity, will be achieved before the hyperbolic curve becomes asymptotic i.e. before the onset of achieving asymptotic value, $\left(\sigma_{\mathrm{rr}}-\sigma_{\theta \theta}\right)_{\mathrm{asymp}}$, it is customary to require that $\left(\sigma_{\mathrm{rr}}-\sigma_{\theta \theta}\right)_{\mathrm{f}}$ is related to $\left(\sigma_{\mathrm{rr}}-\right.$ $\left.\sigma_{\theta \theta}\right)_{\text {asymp }}$ by failure factor, $R_{\mathrm{f}}$, (Duncan and Chang, 1970):

$$
R_{\mathrm{f}}=\text { failure ratio }=\frac{\left(\sigma_{1}-\sigma_{3}\right)_{\mathrm{f}}}{\left(\sigma_{1}-\sigma_{3}\right)_{\text {asymp }}}=\frac{\left(\sigma_{\mathrm{rr}}-\sigma_{\theta \theta}\right)_{\mathrm{f}}}{\left(\sigma_{\mathrm{rr}}-\sigma_{\theta \theta}\right)_{\text {asymp }}}
$$

\section{MODULUS OF ELASTICITY AT VARIOUS LEVELS OF STRESS}

Hyperbolic model as defined by Eq. (8) is shown by curve $\mathrm{ABCD}$ in Fig. 1. At Point $\mathrm{A}, \varepsilon_{\theta \theta}$ equals zero and the
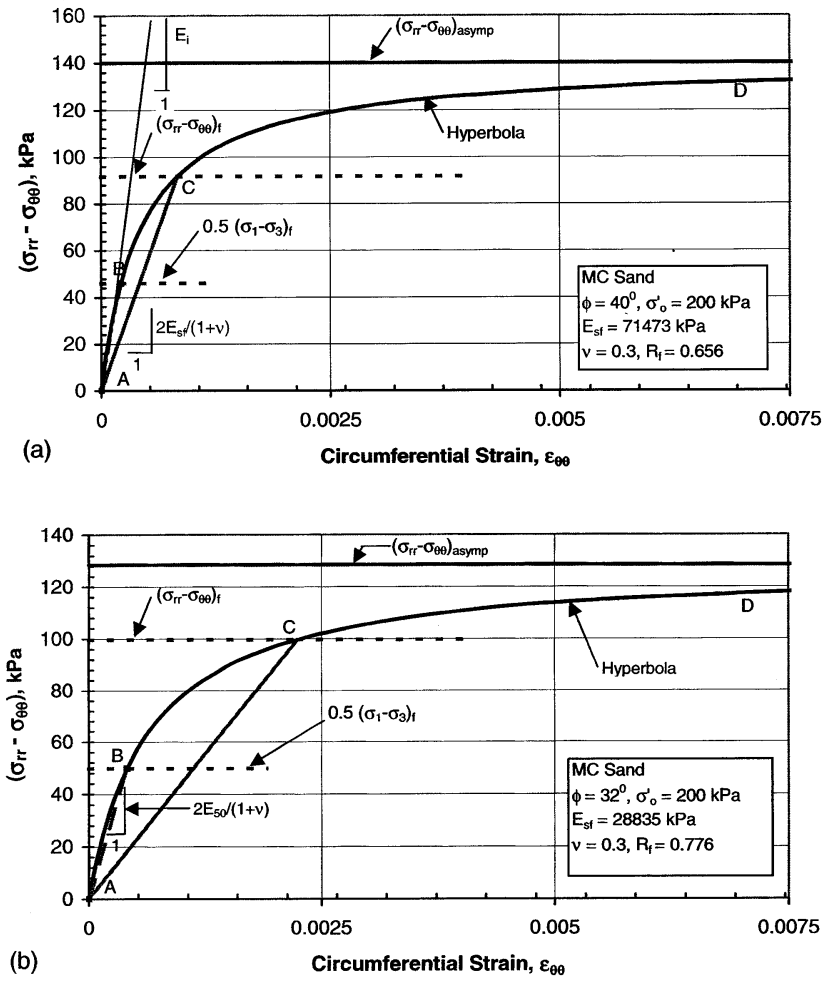

Fig. 1. During cylindrical cavity expansion in medium compressibility (MC) sand, hyperbolic model for stress-strain relationship, at $\sigma^{\prime}{ }_{0}=200 \mathrm{kPa}$, (a) $\phi=40^{\circ}$ and (b) $\phi=32^{\circ}$

tangent to the hyperbolic curve at this point represents initial modulus, $E_{\mathrm{i}}$. At Point $\mathrm{C}$, peak stresses, $\left(\sigma_{\mathrm{rr}}-\sigma_{\theta \theta}\right)_{\mathrm{f}}$, that cause Mohr circle to touch Coulomb-Mohr envelope, are developed and therefore Point $\mathrm{C}$ represents $\left(\sigma_{\mathrm{rr}}-\sigma_{\theta \theta}\right)_{\mathrm{f}}$ as ordinate and $\varepsilon_{\theta \theta}$ of real soil at that instant as abscissa. Figure 2 represents state of stresses and Mohr circles during cylindrical cavity expansion. In Fig. 2, Mohr circle 1 represents the stresses at $0.5\left(\sigma_{\mathrm{rr}}-\sigma_{\theta \theta}\right)_{\mathrm{f}}$, whereas Mohr circle 2 represents the stresses at $\left(\sigma_{\mathrm{rr}}-\sigma_{\theta \theta}\right)_{\mathrm{f}}$ and therefore this circle touches Mohr-Coulomb's failure envelope. Point B in Fig. 1 represents $0.5\left(\sigma_{\mathrm{rr}}-\sigma_{\theta \theta}\right)_{\mathrm{f}}$ as ordinate and $\varepsilon_{\theta \theta}$ value of real soil exhibiting nonlinear response as abscissa at that level of stresses. Similarly, Point $\mathrm{C}$ in Fig. 1 represents $\left(\sigma_{\mathrm{rr}}-\sigma_{\theta \theta}\right)_{\mathrm{f}}$ as ordinate and $\varepsilon_{\theta \theta}$ value of real soil exhibiting nonlinear response as abscissa at that level of stresses. The cavity expansion analysis made in the companion paper (Gupta, 2005) uses linear Path AC for linear stress-strain relationship in place of nonlinear Path ABC of real soil. Slope of Path AC represents secant modulus at failure, $E_{\text {sf }}$. With this view, it is assumed that $E$ used and determined in the companion paper (Gupta, 2005) represents secant modulus at failure, $E_{\mathrm{sf}}$. The slope of Path $\mathrm{AB}$ represents secant modulus at $50 \%$ failure stresses $\left(E_{50}\right)$. Path CD of Fig. 1 represents extension of the hyperbolic curve beyond Point $\mathrm{C}$, to show that if failure conditions represented by peak stresses had not reached at Point $\mathrm{C}$, stress-strain relationship would have continued to be represented by hyperbolic path CD.

It may be noted that the laboratory tests have shown 


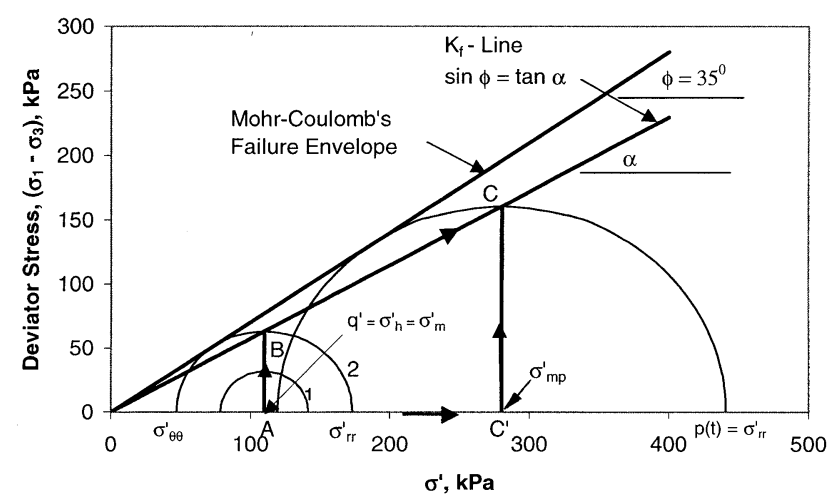

Fig. 2. During cylindrical cavity expansion, stress path, states of stresses and Mohr-Coulomb envelope

that at any state of stress, strain is not dependent upon the stress path (i.e. linear or nonlinear) between the two points, but depends on stresses at the initial such as $\mathrm{A}$ and the subsequent point of the stress path such as B or C, under consideration (Lambe and Whitman, 1969; Vesic, 1972).

As shown below, using the above concept, values of $E_{50}$ and $E_{\mathrm{i}}$ can be determined by using hyperbolic model as defined by Eq. (8). Using $E=E_{\text {sf }}$, when the state of equilibrium is reached at the face of cavity or at the interface of elastic and plastic zone, such as at point $\mathrm{C}$ in Fig. 1, circumferential stress can be written as (Gupta, 2005):

$$
\varepsilon_{\theta \theta}=\varepsilon_{\theta \mathrm{p}}=\frac{(1+v)}{2 E_{\mathrm{sf}}}\left(\sigma_{\mathrm{rr}}-\sigma_{\theta \theta}\right)_{\mathrm{f}}=\frac{(1+v) q^{\prime} \sin \phi}{E}
$$

Where $\varepsilon_{\theta \mathrm{p}}$ denotes circumferential strain at the interface of the elastic and plastic zones or at the face of cavity when the state of equilibrium is reached for the first time. For Point C of Fig. 1, the expression as given in Eq. (10) for $\varepsilon_{\theta \theta}$ i.e. for $\varepsilon_{\theta \mathrm{p}}$, and the expression as given in Eq. (3) for $\left(\sigma_{\mathrm{rr}}-\sigma_{\theta \theta}\right)$ i.e. $\left(\sigma_{\mathrm{rr}}-\sigma_{\theta \theta}\right)_{\mathrm{f}}$ can be substituted in Eq. (9); and after rearranging terms, $E_{\mathrm{sf}}$ or $R_{\mathrm{f}}$ are given by:

$$
E_{\mathrm{sf}}=\frac{E_{\mathrm{i}}\left(1-R_{\mathrm{f}}\right)(1+v)}{2}
$$

or

$$
R_{\mathrm{f}}=1-\frac{2 E_{\mathrm{sf}}}{E_{\mathrm{i}}(1+v)}
$$

Similarly, the relationship between $E_{\mathrm{i}}$ and $E_{50}$ can also be determined. For Point B of Fig. 1, $\varepsilon_{\theta \theta}$ at $0.5\left(\sigma_{1}-\sigma_{3}\right)_{\mathrm{f}}$ is given by (Gupta, 2005):

$$
\varepsilon_{\theta \theta}=\varepsilon_{\theta \theta(0.5 \mathrm{f})}=\frac{1+v}{E_{50}} \frac{\left(\sigma_{\mathrm{rr}}-\sigma_{\theta \theta}\right)_{\mathrm{f}}}{4}
$$

As stated before, $E_{50}$, represented by linear Path AB of Fig. 1, is secant modulus at half of the value of $\left(\sigma_{\mathrm{rr}}-\right.$ $\left.\sigma_{\theta \theta}\right)_{\mathrm{f}}$. Substituting $\left(\sigma_{\mathrm{rr}}-\sigma_{\theta \theta}\right)=0.5 \quad\left(\sigma_{\mathrm{rr}}-\sigma_{\theta \theta}\right)_{\mathrm{f}}$ and the above value of $\varepsilon_{\theta \theta(0.5 \mathrm{f})}$ in Eq. (9) and arranging terms, following expressions for $E_{50}$ in terms of $E_{\mathrm{i}}$ or $E_{\mathrm{sf}}$ are obtained:

$$
E_{50}=0.5(1+v)\left(1-0.5 R_{\mathrm{f}}\right) E_{\mathrm{i}}
$$

or

$$
E_{50}=\frac{\left(1-0.5 R_{\mathrm{f}}\right) E_{\mathrm{sf}}}{\left(1-R_{\mathrm{f}}\right)}
$$

It may be noted that $E_{50}$ has been considered to be approximately equal to modulus determined from unload-reload cycle during SBPM tests (Wroth, 1984). It can also be seen that relationships between $E_{\mathrm{sf}}$ and $E_{\mathrm{i}}$ and between $E_{\mathrm{sf}}$ and $E_{50}$ or between $E_{50}$ and $E_{\mathrm{i}}$ are dependent on the value of failure factor $R_{\mathrm{f}}$. These relationships can only be used when the value of $R_{\mathrm{f}}$ is known.

\section{$E_{\mathrm{sf}}, E_{\mathrm{i}}$ and FAILURE FACTOR, $\boldsymbol{R}_{\mathrm{f}}$}

As shown below, it is possible to estimate $E_{\text {sf }}$ using the existing correlations and $E_{\mathrm{i}}$ using wave equation theory and then value of $R_{\mathrm{f}}$ can be estimated using Eqs. (11b) or (13). For normally consolidated sands (Schmertmann, 1978; Mayne, 2001; Baldi et al., 1989), secant modulus $\left(E_{\mathrm{sf}}\right)$ or constrained modulus $(M)$ can be estimated based on correlations developed between angle of friction, relative density $\left(D_{\mathrm{R}}\right)$, cone tip resistance $\left(q_{\mathrm{c}}\right)$, effective overburden stress $\left(\sigma^{\prime}{ }_{\mathrm{o}}\right)$, effective octahedral stress $\left(\sigma^{\prime}{ }_{\mathrm{oct}}\right)$, and atmospheric pressure $\left(P_{\mathrm{a}}\right)$ as given below:

$\phi=28.0+14\left(D_{\mathrm{r}} / 100\right)$

for high compressibility sand (HC)

$\phi=31.5+11.5\left(D_{\mathrm{r}} / 100\right)$

for medium compressibility sand (MC)

$\phi=34.5+10.0\left(D_{\mathrm{r}} / 100\right)$

$$
\text { for low compressibility sand (LC) }
$$

From Schmertmann (1978)

$$
\phi=17.6+11.0 \log \left(\frac{q_{\mathrm{c}}}{\left(\sigma^{\prime}{ }_{\mathrm{o}} P_{\mathrm{a}}\right)^{0.5}}\right)
$$

From Mayne (2001)

$$
\frac{M}{q_{\mathrm{c}}}=14.48\left(\frac{P_{\mathrm{a}}}{100}\right)\left(\frac{\sigma^{\prime}{ }_{\text {oct }}}{P_{\mathrm{a}}}\right)^{-0.116} e^{-1.123 D_{\mathrm{r}}}
$$

From Baldi et al. (1989)

Using Eqs. (15), (14a), (14b), and (14c), it can be shown that terms $q_{\mathrm{c}}$ and $D_{\mathrm{r}}$ can be eliminated from Eq. (16), to express $M$ in terms of $\phi, P_{\mathrm{a}}, \sigma^{\prime}{ }_{\mathrm{o}}$ and $\sigma^{\prime}$ oct, by the following:

$$
\begin{aligned}
M= & {\left[\left(\sigma^{\prime}{ }_{\mathrm{o}} P_{\mathrm{a}}\right)^{0.5} 10^{\frac{(\phi-17.6)}{11}}\right] 14.48\left(\frac{P_{\mathrm{a}}}{100}\right) } \\
& \times\left(\frac{\sigma^{\prime}{ }_{\mathrm{oct}}}{P_{\mathrm{a}}}\right)^{-0.116} e^{\frac{-1.123(\phi-28)}{14}} \text { for HC Sand } \\
M= & {\left[\left(\sigma_{\mathrm{o}}^{\prime} P_{\mathrm{a}}\right)^{0.5} 10^{\frac{(\phi-17.6)}{11}}\right] 14.48\left(\frac{P_{\mathrm{a}}}{100}\right) } \\
& \times\left(\frac{\sigma^{\prime}{ }_{\mathrm{oct}}}{P_{\mathrm{a}}}\right)^{-0.116} e^{\frac{-1.123(\phi-31.5)}{11.5}} \text { for MC Sand }
\end{aligned}
$$




$$
\begin{aligned}
M= & {\left[\left(\sigma^{\prime}{ }_{\mathrm{o}} P_{\mathrm{a}}\right)^{0.5} 10^{\frac{(\phi-17.6)}{11}}\right] 14.48\left(\frac{P_{\mathrm{a}}}{100}\right) } \\
& \times\left(\frac{\sigma^{\prime}{ }_{\text {oct }}}{P_{\mathrm{a}}}\right)^{-0.116} e^{\frac{-1.123(\phi-34.5)}{10}} \text { for LC Sand }
\end{aligned}
$$

Where: $q_{\mathrm{c}}, E, M, P_{\mathrm{a}},{\sigma^{\prime}}^{\prime}, \sigma^{\prime}{ }_{\text {oct }}$ are in $\mathrm{kPa}$. Relative density, $D_{\mathrm{R}}$ is in percentage. $v$ is Poisson's ratio. $\gamma_{\mathrm{t}}$ is in $\mathrm{kN} / \mathrm{m}^{3} . V_{\mathrm{s}}$ is in $\mathrm{m} / \mathrm{s}$. Depth, $z$ is in $m . E$ and $M$ are related by well-known equation:

$$
E=E_{\mathrm{sf}}=\frac{M(1+v)(1-2 v)}{(1-v)}
$$

Initial modulus $\left(E_{\mathrm{i}}\right)$ can be estimated based on wave propagation theory. Baldi et al. (1989) performed extensive tests on sand and established a correlation between shear wave velocity $\left(V_{s}\right)$, cone tip resistance $\left(q_{c}\right)$, and effective overburden stress $\left(\sigma^{\prime}{ }_{0}\right)$, as shown in Eq. (19). Similarly, Mayne (2001) performed numerous tests to establish a correlation between shear wave velocity $\left(V_{\mathrm{s}}\right)$ and total unit weight $\left(\gamma_{t}\right)$ as shown in Eq. (20).

$$
V_{\mathrm{s}}=4390\left(q_{\mathrm{c}}\right)^{0.13}\left(\sigma^{\prime}{ }_{\mathrm{o}}\right)^{0.27}
$$

From Baldi et al. (1989)

$$
\gamma_{\mathrm{t}}=8.32 \log \left(V_{\mathrm{s}}\right)-1.61 z
$$

From Mayne (2001)

Using Eq. (15), an equation for $q_{\mathrm{c}}$ in terms of $\phi$ and $\sigma^{\prime}{ }_{\circ}$ can be obtained and substituted in Eq. (19) and after rearranging terms, the relationship for $V_{\mathrm{s}}$ in terms of $\phi$, $P_{\mathrm{a}}$, and $\sigma^{\prime}{ }_{\mathrm{o}}$ becomes:

$$
V_{\mathrm{s}}=4390\left(\sqrt{\sigma^{\prime}{ }_{0} P_{\mathrm{a}}} 10^{\frac{\phi-17.6}{11}}\right)^{0.13}\left(\sigma^{\prime}{ }^{\prime}\right)^{0.27}
$$

Finally, after determining $V_{\mathrm{s}}$ and $\gamma_{\mathrm{t}}$, initial modulus $\left(E_{\mathrm{i}}\right)$ and shear modulus $\left(G_{\mathrm{max}}\right)$ are calculated using following well-known relationship.

$$
E_{\mathrm{i}}=2 G_{\max }(1+v)=2(1+v)\left(\frac{\gamma_{\mathrm{t}}}{g}\right)\left(V_{\mathrm{s}}\right)^{2}
$$

Where $g$ is gravitational acceleration constant and is equal to $9.8 \mathrm{~m} / \mathrm{s}^{2}$. Using above equations, values of $E_{\mathrm{i}}$, $E_{\text {sf }}$ and $E_{50}$ were calculated at $\sigma^{\prime}{ }_{o}$ of 100 and $200 \mathrm{kPa}$, for various values of $\phi$, and the $E_{\mathrm{i}}, E_{\mathrm{sf}}$ and $E_{50}$ versus $\phi$ relationships are obtained as shown in Figs. 3, 4 and 5. It may also be noted that typical values of $E_{\mathrm{sf}}$ or $E_{\mathrm{o}}$ at an arbitrary stress of $100 \mathrm{kPa}$ range from 10 to $25 \mathrm{MPa}$ for loose sand, 20 to $60 \mathrm{MPa}$ for medium dense sand and 60 to $100 \mathrm{MPa}$ for dense sand (Coduto, 1994). It can be shown that these typical values of $E_{\mathrm{o}}$ or $E_{\mathrm{sf}}$ and the values of $E_{\mathrm{sf}}$ calculated from above equations are about in the same range, see Figs. 3, 4 and 5 .

Values of failure factor, $R_{\mathrm{f}}$, which is defined by Eq. (11b), can now be estimated because values of $E_{\mathrm{i}}$ and $E_{\text {sf }}$ can be determined using Eqs. (22) and (18). In Figs. 3, 4 , and 5 , values of $R_{\mathrm{f}}$ at $\sigma^{\prime}{ }_{\circ}$ of 100 and $200 \mathrm{kPa}$, for values of $\phi$ have been shown. In these figures, estimated values of $R_{\mathrm{f}}$ are found to vary from 0.5 for very dense sand to about 0.85 for loose sands. Analyzing the triaxial
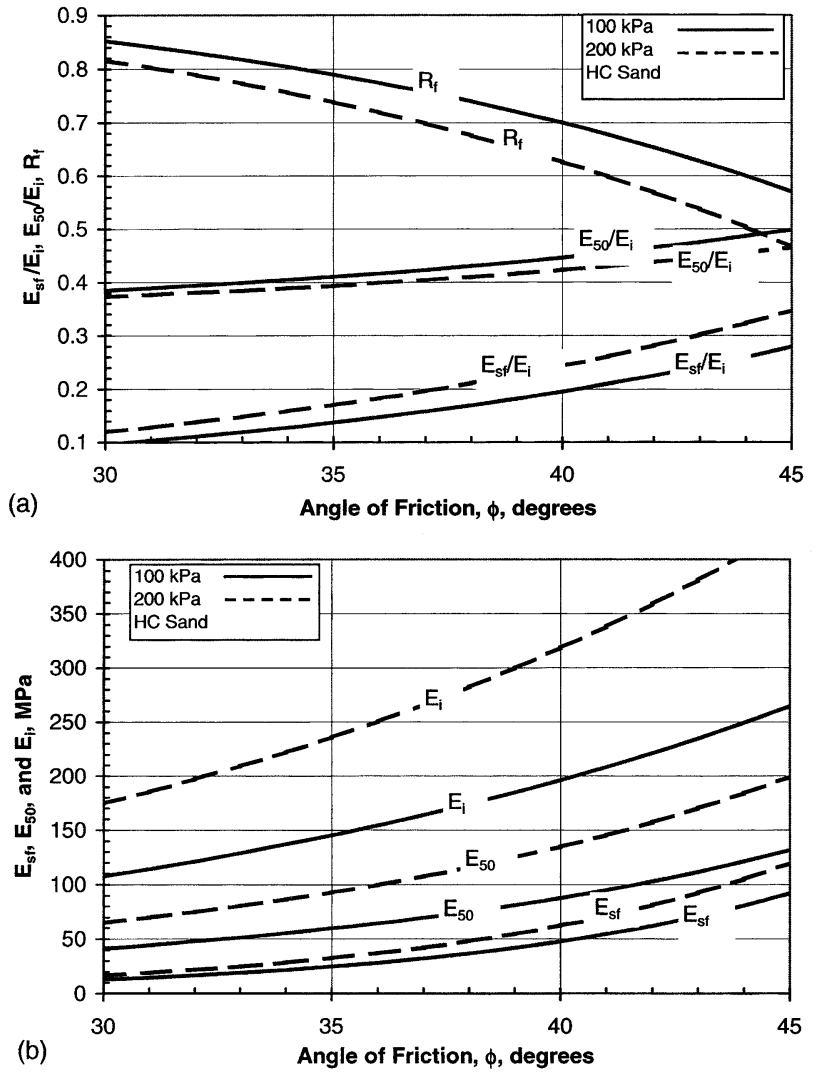

Fig. 3. For high compressibility sand (HC) at effective overburden stress $\left(\sigma^{\prime}{ }_{\mathrm{o}}\right)$ of 100 and $200 \mathrm{kPa}$, (a) Ratios $E_{50} / E_{\mathrm{i}}, E_{\mathrm{sf}} / E_{\mathrm{i}}, R_{\mathrm{f}}$ versus $\phi$ curves and (b) $E_{\mathrm{i}}, E_{50}, E_{\mathrm{sf}}$ versus $\phi$ curves

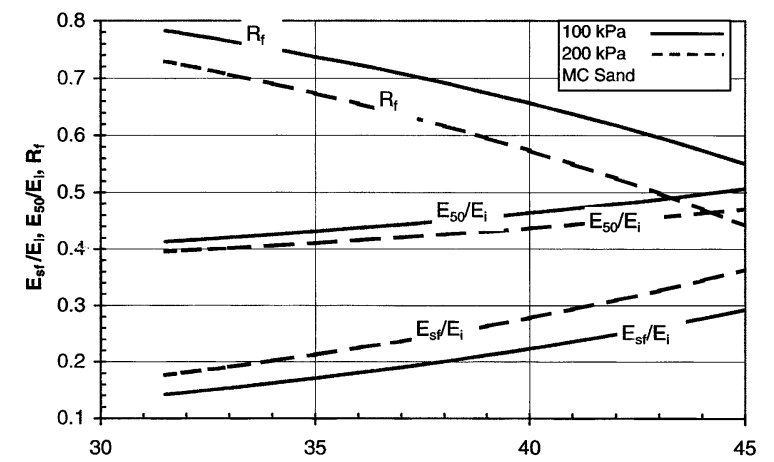

(a)

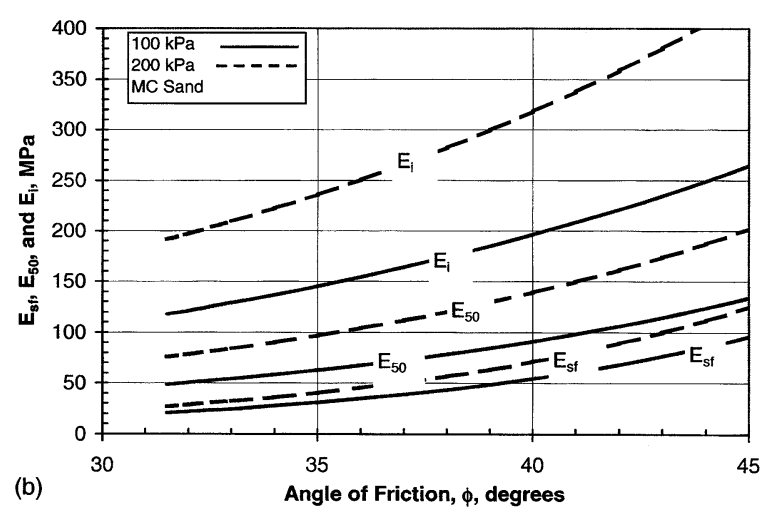

Fig. 4. For medium compressibility sand (MC) at effective overburden stress $\left(\sigma^{\prime}{ }_{\mathrm{o}}\right)$ of 100 and $200 \mathrm{kPa}$, (a) Ratios $E_{50} / E_{\mathrm{i}}, E_{\mathrm{sf}} / E_{\mathrm{i}}, R_{\mathrm{f}}$ versus $\phi$ curves and (b) $E_{\mathrm{i}}, E_{50}, E_{\mathrm{sf}}$ versus $\phi$ curves 

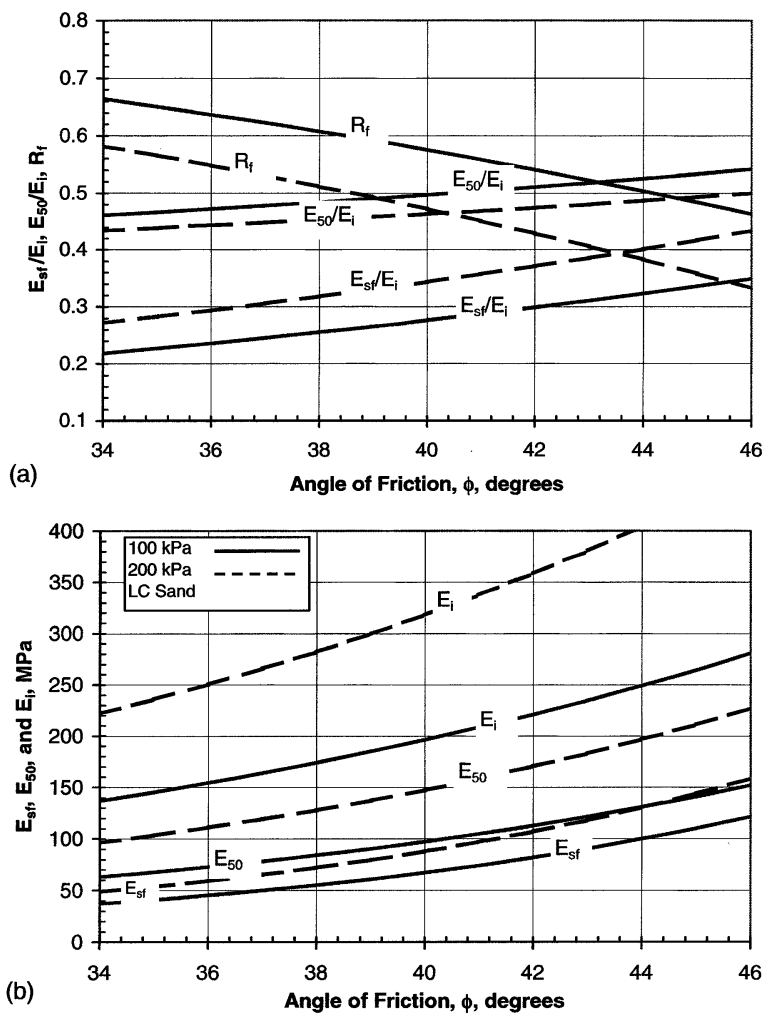

Fig. 5. For low compressibility sand (LC) at effective overburden stress $\left(\sigma^{\prime}{ }_{0}\right)$ of 100 and $200 \mathrm{kPa}$, (a) Ratios $E_{50} / E_{\mathrm{i}}, E_{\mathrm{sf}} / E_{\mathrm{i}}, R_{\mathrm{f}}$ versus $\phi$ curves and (b) $E_{\mathrm{i}}, E_{50}, E_{\mathrm{sf}}$ versus $\phi$ curves

compression tests, Duncan and Chang (1970) had found the value of $R_{\mathrm{f}}$ varying between 0.6 and 0.9 for dense to loose sands.

\section{RELATIONSHIP BETWEEN $F^{\prime \prime}{ }_{q}$ and $\phi$}

Dimensionless cavity expansion factor $\left(F^{\prime \prime}{ }_{q}\right)$ is a variable and relationship between $F^{\prime \prime}{ }_{\mathrm{q}}$ and $\phi$ is dependent on $I_{\mathrm{r}}$ and also on the value of circumferential strain $\left(\varepsilon_{\theta \theta}\right)$, see companion paper (Gupta, 2005). To find a relationship between $F^{\prime \prime}{ }_{q}$ and $\phi$, an effort was made to estimate $F^{\prime \prime}{ }_{q}$ versus $\phi$ curve at a selected value of $\varepsilon_{\theta \theta}$, say at $10 \%$, and also at three selected values of $\sigma^{\prime}$, for normally consolidated sands. For selected type of sand (LC, MC, or HC), the constrained modulus, $M$, can be calculated using Eqs. (17a) or (17b) or (17c), and the secant modulus, $E_{\text {sf }}$ can be calculated using Eq. (18). It can be seen that the value of $E_{\text {sf }}$ determined as above, is dependent only on variables, $\phi, \sigma^{\prime}{ }_{0}$, and $\sigma_{\text {oct }}$. As previously stated, the value of $E_{\mathrm{sf}}$ has been assumed equal to Young's modulus of elasticity, $E$. When value of $E$ based on these equations is substituted in the expression for rigidity index, $I_{\mathrm{r}}$, which is defined by Eq. (23), it can be seen that $I_{\mathrm{r}}$ also becomes dependent on variables, $\phi, \sigma^{\prime}{ }_{\text {o }}$, and $\sigma^{\prime}{ }_{\text {oct }}$. $\sigma^{\prime}$ oct is equal to $\sigma^{\prime}{ }_{0}\left(1+2 K_{0}\right) / 3$, and $K_{0}$ for normally consolidated sand is equal to $(1-\sin \phi)$.

$$
I_{\mathrm{r}}=\frac{E}{2(1+v) q^{\prime} \tan \phi}
$$

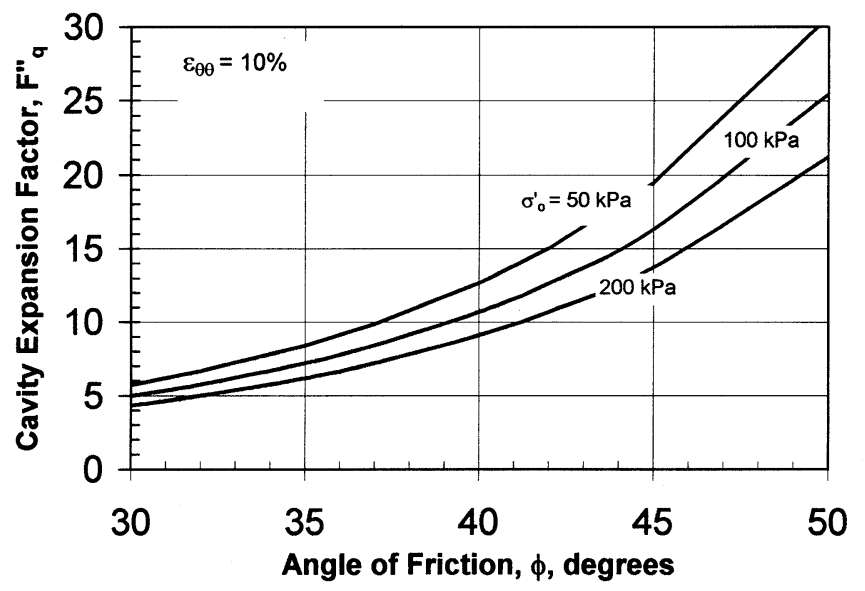

Fig. 6. When circumferential strain at the face of cavity is $\mathbf{1 0} \%$, relationship of cylindrical cavity expansion factor, $F^{\prime \prime}$, with angle of friction and effective overburden stress

where $q^{\prime}=\sigma^{\prime}$.

In this way, for normally consolidated sands, $I_{\mathrm{r}}$ becomes dependent only on $\phi$ and $\sigma^{\prime}$. Therefore, at selected values of $\phi$ and $\sigma^{\prime}{ }_{0}, I_{\mathrm{r}}$ can be calculated, and then value of $F^{\prime \prime}{ }_{q}$ can be calculated at $\varepsilon_{\theta \theta}=10 \%$, using the procedure explained in the companion paper, Gupta (2005). The $F^{\prime \prime}{ }_{q}$ versus $\phi$ curve at $\sigma^{\prime}{ }_{0}=50,100$ and 200 $\mathrm{kPa}$, when $\varepsilon_{\theta \theta}$ is equal to $10 \%$ is shown in Fig. 6 .

\section{BASIS OF ANALYZING SBPMT DATA}

As explained above, at selected values of $\phi$ and $I_{\mathrm{r}}$, dimensionless factor $\left(F^{\prime \prime}{ }_{q}\right)$ can be calculated for various values of circumferential strain $\left(\varepsilon_{\theta \theta}\right)$. Internal pressure, $p(t)$, applied during SBPM test and corresponding increase in radius of cavity, which occurs at applied internal pressure, $p(t)$, is utilized to develop $F^{\prime \prime}{ }_{q}$ versus $\varepsilon_{\theta \theta}$ curve. $F^{\prime \prime}{ }_{\mathrm{q}}$ is equal to $p(t) / \sigma_{{ }_{\mathrm{h}}}$. $\sigma_{{ }_{\mathrm{h}}}$ is measured during a SBPM test. $\varepsilon_{\theta \theta}$ is equal to $\left[\left\{R(t)-R_{\mathrm{i}}\right\} / R_{\mathrm{i}}\right] . R(t)$ is radius of the cavity at time $t$, when internal pressure is $p(t) . R_{\mathrm{i}}$ is the initial radius of the cavity, i.e. initial radius of the SBPM probe prior to application of the internal pressure just above the value of in-situ $\sigma^{\prime}{ }_{\mathrm{h}}$. In this way, $F^{\prime \prime}{ }_{\mathrm{q}}$ versus $\varepsilon_{\theta \theta}$ curve obtained from SBPMT data is compared with theoretical $F^{\prime \prime}{ }_{q}$ versus $\varepsilon_{\theta \theta}$ curve developed at selected values of $I_{\mathrm{r}}$ and $\phi$. Several trials are made, and the value of $I_{\mathrm{r}}$ and $\phi$ that produces a theoretical $F^{\prime \prime}{ }_{q}$ versus $\varepsilon_{\theta \theta}$ curve closely matching with actual $F^{\prime \prime}{ }_{q}$ versus $\varepsilon_{\theta \theta}$ curve obtained from SBPMT is selected. It may be mentioned that because there are two variables, $\phi$ and $I_{\mathrm{r}}$, it becomes difficult to find an optimum set of $\phi$ and $I_{\mathrm{r}}$. This has to be decided based on judgment. Author first determined $F^{\prime \prime}{ }_{q}$ value at $\varepsilon_{\theta \theta}=10 \%$ from the SBPMT data and then at that determined value of $F^{\prime \prime}{ }_{\mathrm{q}}$ and $\sigma^{\prime}{ }_{\mathrm{o}}$ at the depth where SBPM tests was conducted, an approximate value of $\phi$ was determined from Fig. 6. Using this value of $\phi$, and selected values of $I_{\mathrm{r}}$, theoretical $F^{\prime \prime}{ }_{\mathrm{q}}$ versus $\varepsilon_{\theta \theta}$ curve were determined and matched with actual $F^{\prime \prime}{ }_{\mathrm{q}}$ versus $\varepsilon_{\theta \theta}$ curve, obtained from SBPM test. Generally, it was found that 
optimum value of $\phi$ was within \pm 0.5 degrees of $\phi$ determined from Fig. 6. In this way, an optimum set of $\phi$ and $I_{\mathrm{r}}$ was determined. From known values of $\phi$ and $I_{\mathrm{r}}$, the value of $E$ can be determined from Eq. (23). Poisson's ratio, $v$, is generally assumed as 0.3 for SBPM tests.

As already mentioned that the value of $E$ so determined is assumed equal to secant modulus, $E_{\mathrm{sf}}$, at failure, i.e. peak stresses at the state of equilibrium. Once, the value of $E_{\mathrm{sf}}$ is determined, an effort is made to determine the value of $E_{50}$ and $E_{\mathrm{i}}$. With this objective, an approximate value of $R_{\mathrm{f}}$ is estimated from Figs. 3, or 4 or 5, based on estimated value of $\phi$ and known value of $\sigma^{\prime}{ }_{0}$. Knowing the value of $R_{\mathrm{f}}$, the value of $E_{50}$ can be determined from Eq. (13b) and value of $E_{\mathrm{i}}$ from Eq. (11a). On this basis, SBPMT data of several sites were analyzed to determine values of $\phi, E_{\mathrm{sf}}$, and $E_{50}$, as described hereunder.

\section{EXAMPLES}

SBPM tests in sands have been performed worldwide. Some published test data/results of SBPM tests performed by Bruzzi et al. (1986), Houlsby and Clark (1986), Robertson and Hughes (1986) and Wroth (1984), is analyzed, using the method described in this paper.

\section{Po River Sand}

SBPM test data of Bruzzi et al. (1986) was analyzed and the analyzed results for a SBPM test performed at a depth of $17.9 \mathrm{~m}$ in Po river medium to coarse sand, are shown in Fig. 7(a) and Table 1. The depth to water table was $0.5 \mathrm{~m}$. The values of $\gamma_{\mathrm{T}}, \sigma^{\prime}{ }_{\mathrm{o}}, \phi$, and shear modulus $\left(G_{\text {ur }}\right)$ estimated from unload-reload cycles by Bruzzi et al. (1986) are shown in this table. The coefficient of earth pressure at rest $\left(K_{\mathrm{o}}\right)$ was stated to be equal to $0.65 \pm 0.18$. SBPMT data consisted of a curve of internal cavity pressure, $p(t)$, versus radial strain. Dividing $p(t)$ by $q^{\prime}$, $F^{\prime \prime}{ }_{q}$ versus $\varepsilon_{\theta \theta}$ curve was obtained as shown in Fig. 7(a). An excellent match was found between measured data and the theoretical curve derived using the above method when values of $I_{\mathrm{r}}, \phi$, and $K_{\mathrm{o}}$ were selected as $300,38^{\circ}$ and 0.725 , respectively. Based on values of $\phi$ and $\sigma^{\prime}{ }_{0}$, approximate value of $R_{\mathrm{f}}$ as 0.68 was selected from Fig. 3. The values of $E_{\mathrm{sf}}, E_{50}$ and $E_{\mathrm{i}}$ were then calculated using
Eqs. (23) and (13b), and 11(a), respectively as shown in Table 1. Value of $E_{50}$ compares well with value of $E_{\mathrm{r}}$ determined from unload/reload cycles. The value of $E_{\text {sf }}$ compares well with typical values of $E_{\text {sf }}$ for dense sands. The value of $\phi$ selected as $38^{\circ}$ matches with that estimated by Bruzzi et al. (1986).

\section{Shotter Paper Mill Sand}

Houlsby et al. (1986) performed SBPM tests at a depth of $5.8 \mathrm{~m}$ in uniformly graded fine to coarse sand at the site of Shotter Paper Mill, North Wales, U.K. The ground water level was at a depth of $3.8 \mathrm{~m}$. Houlsby et al. (1986) stated the value of $\gamma, \sigma^{\prime}{ }_{0}, K_{0}$, and $\phi$ as $18 \mathrm{kN} / \mathrm{m}^{3}$, $84.8 \mathrm{kPa}, 0.9$ and 38 degrees, respectively. A good match between measured curve and theoretical curve derived at $I_{\mathrm{r}}=380, K_{\mathrm{o}}=0.9$ and $\phi=38^{\circ}$ was obtained as shown in
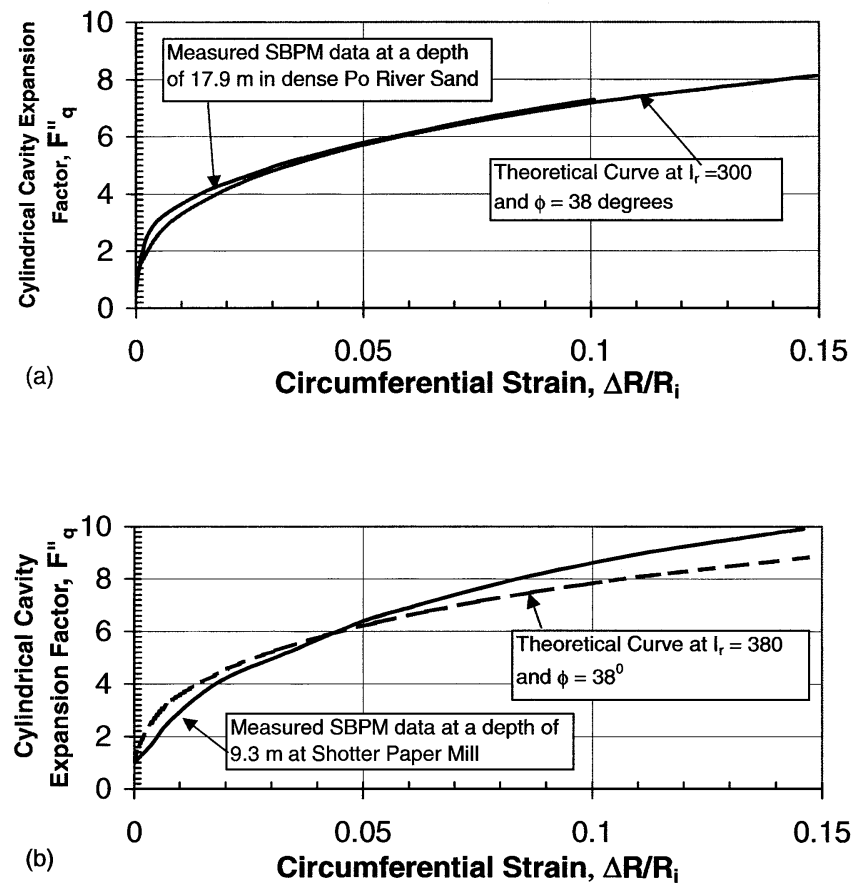

Fig. 7. Comparison of $\mathbf{F}^{\prime \prime}{ }_{q}$ versus $\varepsilon_{\theta \theta}$ curves determined from SBPMT data with theoretical curve for (a) Po River Sand (Bruzzi et al., 1986) and (b) Shotter Paper Mill Sand (Houlsby et al., 1986)

Table 1. From SBPM tests in sands, $\phi$ and $E_{\mathrm{sf}}$ determined using finite strain analysis for cavity expansion (Gupta, 2005) and then, $E_{50}$ and $E_{\mathrm{i}}$ determined using hyperbolic model

\begin{tabular}{|c|c|c|c|c|c|c|c|c|c|c|c|c|c|c|}
\hline Description & Depth & $\begin{array}{c}\text { Depth to } \\
\text { water } \\
\text { table }\end{array}$ & $\gamma_{\mathrm{t}}$ & $\sigma^{\prime}{ }_{0}$ & $K_{\mathrm{o}}$ & $\phi$ & $q^{\prime}$ & $I_{\mathrm{r}}$ & $E_{\text {sf }}$ & $R_{\mathrm{f}}$ & $E_{50}$ & $E_{\mathrm{i}}$ & $G_{\mathrm{ur}}$ & $E_{\mathrm{r}}$ \\
\hline & $\mathrm{m}$ & $\mathrm{m}$ & $\mathrm{kN} / \mathrm{m}^{3}$ & $\mathrm{kPa}$ & & Deg. & $\mathrm{kPa}$ & & $\mathrm{MPa}$ & & $\mathrm{MPa}$ & $\mathrm{MPa}$ & $\mathrm{MPa}$ & $\mathrm{MPa}$ \\
\hline Po River Sand & 17.9 & 0.5 & 19.4 & 176.74 & 0.725 & 38 & 128.1 & 300 & 78.09 & 0.68 & 161.1 & 375.3 & 61.8 & 160.68 \\
\hline Zeebrughe Sand & 10.6 & 4.3 & 15.1 & 98.32 & 0.52 & 39 & 51.1 & 500 & 53.82 & 0.6 & 94.2 & 206.9 & 40.0 & 96 \\
\hline McDonald's Farm & 10.9 & 2 & 18.85 & 118.24 & 0.83 & 35 & 98.14 & 250 & 44.67 & 0.63 & 82.7 & 185.7 & 33.0 & 85.8 \\
\hline McDonald's Farm & 9.3 & 2 & 18.85 & 103.8 & 1.1 & 40 & 114.1 & 500 & 124.5 & & & & & \\
\hline
\end{tabular}

Note: The values of $\gamma, \sigma_{\mathrm{o}}^{\prime}, K_{\mathrm{o}}, G_{\mathrm{ur}}$ and $E_{\mathrm{r}}$ are from Bruzzi et al. (1986), Wroth (1984), Houlsby et al. (1986), and Robertson and Hughes (1986). 


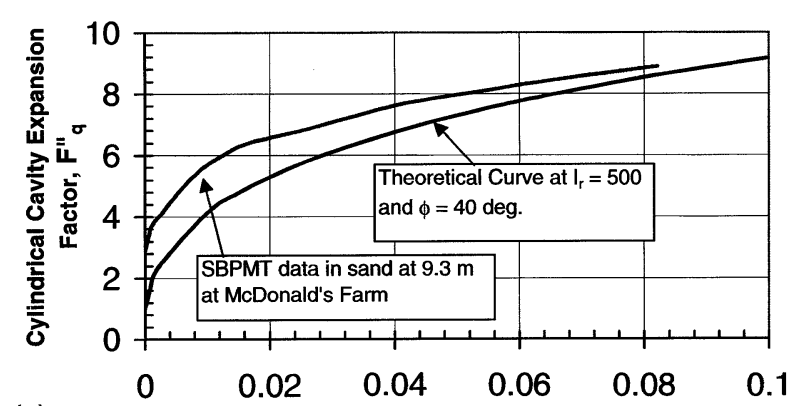

(a)

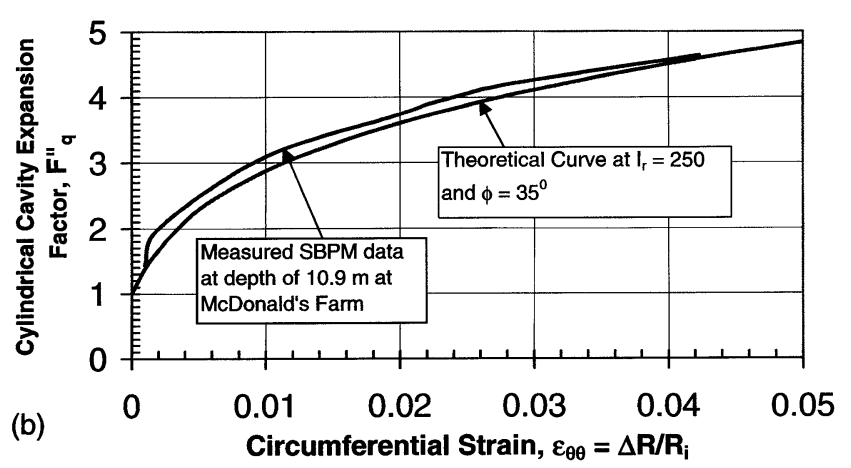

Fig. 8. Comparison of theoretical curves with $F^{\prime \prime}{ }_{q}$ versus $\varepsilon_{\theta \theta}$ curves determined from SBPMT performed in sand at McDonald's Farm (Robertson and Hughes, 1986) at depths (a) $9.3 \mathrm{~m}$ and (b) $10.9 \mathrm{~m}$

Fig. 7(b). An approximate value of $R_{\mathrm{f}}$ as 0.6 was estimated from Fig. 5. As shown in Table 1, the value of $E_{\mathrm{r}}$ calculated from the unload-reload cycle compares well with $E_{50}$, estimated based on $E_{\mathrm{sf}}$ and $R_{\mathrm{f}}$. $\phi$ of $38^{\circ}$ was also determined by Houlsby et al. (1986).

\section{Dense Sand at McDonald's Farm}

Robertson and Campanella (1988) and Robertson and Hughes (1986) have performed cone penetration tests and SBPM tests in dense sand at McDonald's Farm site. The cone tip resistance from 9 to 12 meters varies widely from 15 bars to more than 200 bars, showing either the variability of the density of the sand deposit or the presence of very thin sand-witched layers of saturated cohesive material. Two SBMT tests were performed at depths of 9.3 and $10.9 \mathrm{~m}$ (Robertson and Hughes, 1986). A good match between measured curve and theoretical curve derived at $I_{\mathrm{r}}=500, K_{\mathrm{o}}=1.1$ and $\phi=40^{\circ}$ for the test at $9.3 \mathrm{~m}$ and at $I_{\mathrm{r}}=250, K_{\mathrm{o}}=0.83$ and $\phi=35^{\circ}$ for test at $10.9 \mathrm{~m}$ was obtained as shown in Figs. 8(a) and 8(b). The value $E_{50}$ estimated by the above method, compares well with $E_{\mathrm{r}}$ determined from a reload/unload cycle performed at $10.9 \mathrm{~m}$ depth, see Table 1 .

\section{Zeebrughe Sand}

Wroth (1984) performed a SBPM test in sand at a depth of $10.6 \mathrm{~m}$. The ground water table was at a depth of $4.3 \mathrm{~m}$. $\sigma_{\mathrm{h}}$ was measured as $107 \mathrm{kPa}$ and $\sigma_{\mathrm{o}}$ was estimated as $160 \mathrm{kPa}$ and on that basis $K_{\mathrm{o}}$ was determined as 0.46 . The value of $\phi$ was determined as $39^{\circ}$ and the value of $G_{\mathrm{ur}}$ was estimated as $31 \mathrm{MPa}$, see Wroth (1984). A good match between measured curve and theoretical

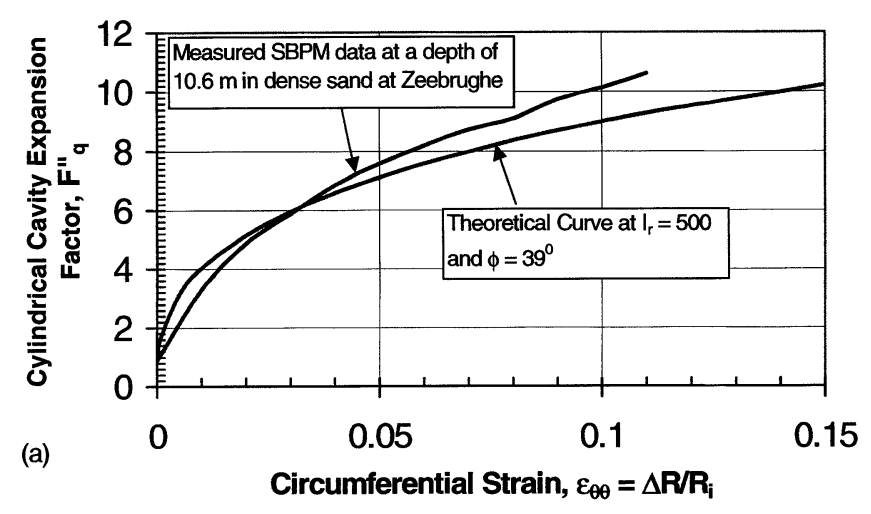

(b)

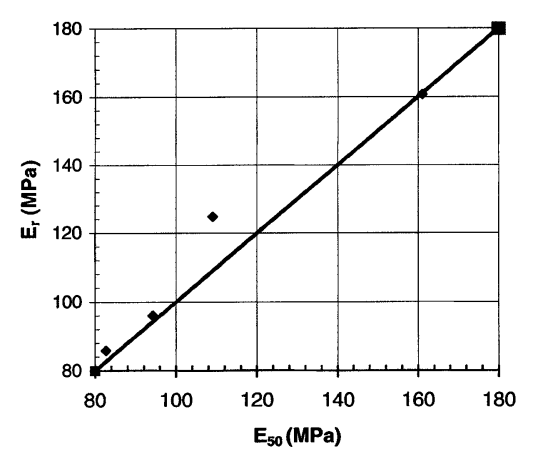

Fig. 9. (a) Comparison of theoretical curves with $F^{\prime \prime}{ }_{q}$ versus $\varepsilon_{\theta \theta}$ curves determined from SBPMT performed in Zeebrugh sand (Wroth, 1984) and (b) Comparison $E_{50}$ estimated by theoretical method with $E_{\mathrm{r}}$ from SBPMT

curve derived at $I_{\mathrm{r}}=500, K_{\mathrm{o}}=0.52$ and $\phi=39^{\circ}$ was obtained as shown in Fig. 9(a). As shown on Table 1, the value of $E_{\mathrm{r}}$ from unload-reload cycle compares well with $E_{50}$ estimated by the above method.

Figure 9(b) shows that $E_{\mathrm{r}}$ estimated from unloadreload cycles compare well with $E_{50}$ for Po river sand, Zeebrughe sand, Shotter paper mill sand and McDonald's Farm sand. These examples show that the SBPM data can be analyzed to determine reasonable values of $\phi$ and modulus at various stress levels $\left(E_{\mathrm{sf}}, E_{50}\right.$ and $E_{\mathrm{i}}$ ), when volumetric strains in the plastic zone are calculated by finite strain method for solving cylindrical cavity expansion.

\section{CONCLUSIONS}

Using cylindrical cavity expansion analysis and estimating volumetric strains by finite strain method, the value of the angle of friction and modulus of deformation at various stress levels can be estimated with reasonable accuracy as demonstrated by the analysis of several SBPM tests in sand performed in various geographical regions. The modulus of deformation $\left(E_{\mathrm{sf}}\right)$, determined from analysis of SBPMT data was found to lie in the range of typical values for that type of sand. The hyperbolic model for nonlinear stress-strain relationship can be successfully used to determine modulus at $50 \%$ failure stresses and also initial tangent modulus by using the 
procedure described in this paper. It is considered that unload/reload modulus determined from SBPMT is related to modulus at 50\% failure stresses (Wroth, 1984). Modulus at $50 \%$ failure stresses determined by using the procedure described in this paper matched well with unload/reload modulus determined from SBPM tests conducted in sand at several sites, demonstrating the reliability and accuracy of this method.

\section{NOTATION}

The following symbols are used in this paper:

$a, b=$ Hyperbolic model parameter;

$D_{\mathrm{R}}=$ Relative density in percent;

$G=$ gravitational acceleration constant;

$E=$ Young's Modulus of Elasticity;

$E_{\text {sf }}=$ Secant Modulus at failure or at state of equilibrium;

$E_{50}=$ Secant Modulus at half value of failure stresses;

$E_{\mathrm{i}}=$ Initial modulus of elasticity;

$E_{\mathrm{r}}=$ Unload $/$ reload modulus from SBPMT;

$E_{\mathrm{o}}=$ Modulus of Elasticity at $\sigma^{\prime}{ }_{\mathrm{o}}=100 \mathrm{kPa}$;

$G=$ shear modulus;

$G_{\max }=$ Initial or maximum shear modulus;

$K_{\mathrm{o}}=$ coefficient of earth pressure at rest;

$h_{\mathrm{w}}=$ height of ground water table above test location;

$I_{\mathrm{r}}=$ rigidity index;

$M=$ Constrained modulus;

$R_{\mathrm{f}}=$ failure factor;

$R_{\mathrm{i}}=$ initial radius of cavity

$r_{\mathrm{p}}=$ radius of plastic zone at time $t$;

$R(t)=$ radius of cavity at time $t$;

$\Delta R=$ change in radius of Cavity, $\left\{R(t)-R_{\mathrm{i}}\right\}$;

$r(t)=$ radial distance of particle from axis of cavity in its displaced position;

$p(t)=$ internal cavity pressure at time $t$;

$q=$ total isotropic ground stress, equal to $\sigma_{\mathrm{h}}$ for cylindrical cavity;

$q^{\prime}=$ effective isotropic ground stress, equal to $\sigma^{\prime}{ }_{\mathrm{h}}$ for cylindrical cavity;

$F^{\prime \prime}{ }_{\mathrm{q}}=$ Dimensionless cylindrical cavity factor at time $t$;

$z=$ depth from ground surface;

$q_{\mathrm{c}}=$ cone penetration resistance;

$V_{\mathrm{s}}=$ shear modulus;

$P_{\mathrm{a}}=$ atmospheric pressure, equal to $101.3 \mathrm{kPa}$;

$\nu=$ Poisson's ratio;

$\gamma_{\mathrm{w}}=$ unit weight of water;

$\gamma_{\mathrm{t}}=$ total unit weight of soil;

$\varepsilon_{\mathrm{rr}}=$ radial strain;

$\varepsilon_{\theta \theta}=$ circumferential strain in cylindrical coordinates;

$\varepsilon_{\theta \mathrm{p}}=$ circumferential strain at interface of elastic and plastic zones;

$\varepsilon_{\theta \theta(0.5 \mathrm{f})}=$ circumferential strain at $50 \%$ failure stresses;

$\varepsilon_{\mathrm{v}}=$ volumetric strain

$\varepsilon_{\mathrm{v} 1}=$ volumetric strain in Path $\mathrm{AC}$;

$\varepsilon_{\mathrm{v} 2}=$ volumetric strain in Path $\mathrm{C}^{\prime} \mathrm{C}$;

$d V=$ volume of an element of width $d r$ and unit height;

$d r=$ width of an element at radial distance $r(t)$;

$\xi_{\mathrm{r}}=$ radial displacement of particle at any radial distance;

$\xi_{\mathrm{rc}}=$ radial displacement of particle at face of cavity;

$\xi_{\mathrm{rp}}=$ radial displacement of particle at interface of elastic and plastic zones;

$\sigma_{\mathrm{rr}}=$ total radial stress;

$\sigma_{\theta \theta}=$ total circumferential stress in cylindrical coordinates;

$\sigma^{\prime}{ }_{r \mathrm{r}}=$ effective radial stress;

$\sigma_{\theta \theta}^{\prime}=$ effective circumferential stress in cylindrical coordinates;

$\sigma^{\prime}{ }_{z z}=$ normal stress in vertical direction; $\sigma_{\mathrm{h}}=$ total horizontal stress;

$\sigma^{\prime}{ }_{h}=$ effective horizontal stress;

$\sigma^{\prime}{ }^{\prime}=$ effective vertical stress;

$\sigma^{\prime}{ }_{\mathrm{m}}=$ mean effective horizontal stress in elastic zone;

$\sigma_{\mathrm{mp}}^{\prime}=$ mean effective horizontal stress in plastic zone;

$\sigma_{\mathrm{m}}=$ mean horizontal stress in elastic zone;

$\sigma^{\prime}{ }_{\text {oct }}=$ effective octahedral stress in elastic zone;

$\sigma_{\text {oct }}=$ total octahedral stress;

$\sigma_{\text {rp }}^{\prime}=$ effective radial stress at interface of elastic and plastic zones;

$\sigma_{\theta \mathrm{p}}^{\prime}=$ effective circumferential stress at interface of elastic and plastic zones;

$\left(\sigma_{\mathrm{rr}}-\sigma_{\theta \theta}\right)_{\text {asymp }}=$ asymptotic value of $\left(\sigma_{\mathrm{rr}}-\sigma_{\theta \theta}\right)$ in hyperbolic model;

$\left(\sigma_{\mathrm{rr}}-\sigma_{\theta \theta}\right)_{\mathrm{f}}=$ Peak stresses at which Mohr circle touches MohrCoulomb failure envelope, or failure stresses;

$\left(\sigma_{\mathrm{rr}}-\sigma_{\theta \theta}\right)_{0.5 \mathrm{f}}=50 \%$ of failure stresses;

$\Delta=$ average volumetric strain in plastic region, dimensionless;

$\phi=$ angle of shearing resistance of soil.

\section{REFERENCES}

1) Baguelin, F., Jezequel, J-F. and Shields, D. H. (1978): The pressuremeter and foundation engineering, Trans. Tech. Publications, Clausthal, Germany, 617.

2) Baldi, G., Bellotti, R., Ghionna, V. N., Jamiolkowski, M. and LoPresti, D. C. F. (1989): Modulus of sands from CPTs and DMTs, Proc. 12th ICSMFE, Rio de Janeiro, Balkema/Rotterdam, 1, 165-170.

3) Bruzzi, D., Ghionna, V., Jamiolkowski, M., Lancelotta, R. and Manfredini, G. (1986): Self-boring pressuremeter tests in Po river sand, ASTM Special Technical Publication 950, The Pressuremeter and its Marine Applications: Second International Symposium, $57-74$.

4) Coduto, D. P. (1994): Foundation Design, Principles and Practices, Prentice Hall, Englewood, NJ, 796.

5) Desai, C. S. and Christian, J. T. (1977): Numerical Methods in Geotechnical Engineering, McGraw-Hill Book Company, New York, 783.

6) Duncan, J. M. and Chang, C. Y. (1970): Nonlinear analysis of stress strain in soils, J. Geotech. Engrg., ASCE, 96 (5), 1629-1653.

7) Gupta, R. C. (2005): Finite strain analysis for self-boring pressuremeter tests in sand, Soils and Foundations, 45(5), 73-79.

8) Houlsby, G. T., Clark, B. G. and Wroth, C. P. (1986): Analysis of the unloading of a pressuremeter in sand, ASTM Special Technical Publication 950, The Pressuremeter and its Marine Applications: 2nd International Symposium, 245-262.

9) Kondner, R. L. (1963): Hyperbolic stress-strain response: Cohesive soils, J. Soil Mech. Found. Div., ASCE, 89 (SM1), 115-143.

10) Lambe, T. W. and Whitman, R. V. (1969): Soil Mechanics, John Wiley and Sons, New York, 553.

11) Mayne, P. W. (2001): Stress-strain-strength-flow parameters from enhanced in-situ tests, Proc. Inter. Conf. In-situ Measurements of Soil Properties and Case Histories, Bali, Indonesia, 27-48.

12) Robertson, P. K. and Hughes, M. O. (1986): Determination of properties of sand from self-boring pressuremeter tests, ASTM Special Technical Publication 950, The Pressuremeter and its Marine Applications: Second International Symposium, 283-302.

13) Robertson, P. K. and Campanella, R. G. (1988): Guidelines for using CPT, CPTU and Marchetti DMT for Geotechnical Design, FHWA-PA-87-023 + 84-24, FHWA, Washington, D. C.

14) Schmertmann, J. H. (1978): Guidelines for Cone Penetration Test: Performance and Design, FHWA-TS-209, FHWA, Washington, D. C.

15) Vesic, A. S. (1972): Expansion of cavities in infinite soil Mass, $J$. Soil Mech. Found. Div., ASCE, 98 (3), 265-290.

16) Wroth, C. P. (1984): The interpretation of in situ soil tests, Geotechnique, 34 (4), 449-489. 